\title{
Inter-relation between Altered Nutritional Status and Clinical Outcomes in Patients with Acute Myocardial Infarction Admitted in a Tertiary Intensive Cardiac Care Unit
}

\author{
Victoria Rus ${ }^{1}$, Diana Opincariu ${ }^{2,3}$, Roxana Hodas ${ }^{2,3}$, Tiberiu Nyulas ${ }^{2}$, Marian Hintea $^{1}$, \\ Theodora Benedek ${ }^{2,3}$ \\ ${ }^{1}$ Department of Nutritional Diseases, University of Medicine and Pharmacy, Tîrgu Mureș, Romania \\ 2 Department of Acute Cardiac care, Clinic of Cardiology, County Clinical Emergency Hospital, Tîrgu Mureș, Romania \\ ${ }^{3}$ Department of Internal Medicine, Clinic of Cardiology, University of Medicine and Pharmacy, Tîrgu Mureș, Romania
}

\begin{abstract}
Background: The impact of nutritional status on the early outcome of subjects with acute myocardial infarction (AMI) is still not completely elucidated. This study aimed to assess the correlation between nutritional status, as expressed by the CONUT and PIN scores, and (1) clinical and laboratory characteristics, (2) complication rates, and (3) length of hospitalization, in patients with AMI. Materials and methods: We included 56 consecutive patients with AMI who underwent primary percutaneous intervention and stenting. Evaluation of the nutritional status was comprised in the calculation of the CONUT and PNI scores. The study population was divided into 2 groups according to the calculated CONUT score, as follows: group 1 - CONUT score $<3$ points (normal to mildly impaired nutritional status) and group 2 - CONUT score $\geq 3$ points (moderate to severe malnutrition). The primary end-point of the study was the rate of in-hospital complications (left ventricular free wall rupture, hemodynamic instability requiring inotropic medication, high-degree atrioventricular block, the need for temporary cardiostimulation, supraventricular and ventricular arrhythmias and in-hospital cardiac arrest). The secondary end-points included the duration of hospitalization and the length of stay in the intensive cardiac care unit. Results: In total, 56 patients (44.64\% with STEMI, 55.35\% with NSTEMI) with a mean age of $61.96 \pm 13.42$ years, $58.92 \%$ males were included in the study. Group distribution was: group $1-76.78 \%(n=43)$, group $2-23.21 \%(n=23)$. There were no differences between the two groups regarding age, gender, cardiovascular risk factors, or comorbidities. PNI index in group 1 was 54.4 \pm 10.4 and in group $241.1 \pm 2.8, \mathrm{p}<0.0001$. Serum albumin was significantly lower in group $1-4.1 \pm 0.3 \mathrm{vs}$. group $2-3.6 \pm 0.3(\mathrm{p}<0.0001$ ), similarly to total cholesterol levels (group $1-194.9 \pm 41.5$ vs. group $2-161.2$ $\pm 58.2, \mathrm{p}=0.02)$. The complete blood cell count showed that group 2 presented lower levels of hematocrit $(\mathrm{p}=$ 0.003), hemoglobin ( $\mathrm{p}=0.002$ ), and lymphocytes ( $<$ <.0001) compared to group 1 , but a significantly higher platelet count $(\mathrm{p}=0.001)$, mean platelet volume $(\mathrm{p}=0.03)$, neutrophil/lymphocyte $(\mathrm{p}<0.0001)$ and platelet/ lymphocyte $(\mathrm{p}<0.0001)$ ratios, indicating enhanced blood thrombogenicity and inflammation. Regarding in-hospital complications, group 2 presented a higher rate of hemodynamic instability (group $1-11.6 \%$ vs. group $2-38.4 \%, \mathrm{p}=0.02$ ). The overall hospitalization period was $7.7 \pm 1.4$ days in group 1 vs. $10.2 \pm 4.8$ days in group 2, $\mathrm{p}=0.06$; while the duration of stay in the intensive cardiac care unit was $2.6 \pm 0.5$ days in group 1 vs. $4.0 \pm 2.5$ days in group $2, p=0.02$. Conclusions: This study proved that nutritional deficit in acute myocardial patients who undergo revascularization is associated with an increased rate of in-hospital complications and with a longer observation time in a tertiary intensive cardiac care unit.
\end{abstract}

Keywords: nutritional status, myocardial infarction, short-term complications, CONUT score

\section{ARTICLE HISTORY}

Received: January 21, 2018

Accepted: February 26, 2018

\section{CORRESPONDENCE}

Diana Opincariu

Str. Gheorghe Marinescu nr. 38

540139 Tîrgu Mureș, Romania

Tel: +40265215551

E-mail: diana.opincariu@yahoo.ro 


\section{INTRODUCTION}

According to the Third Global Myocardial Infarction Task Force, acute myocardial infarction (AMI) has been defined as necrosis of the cardiac cells triggered by extended ischemia that leads to specific clinical, electrocardiographic, and laboratory changes. ${ }^{1}$ The clinical correspondent of an AMI typically includes non-localized chest pain usually lasting for more than 20 minutes, with specific radiation sites and associated shortness of breath, fatigue and sweating. Although frequently not enough to diagnose an AMI by itself, the 12-lead ECG tracing can promptly identify patients with active myocardial ischemia and can further direct patient management towards an invasive or noninvasive therapeutic approach. Also, ECG recordings can distinguish between ST-elevation myocardial infarction (STEMI), which requires immediate revascularization if under 12 hours from the onset of symptoms, and nonST segment elevation MI (NSTEMI), which presents different timing of revascularization procedures, according to various risk prediction tools. ${ }^{2-4}$

Serum biomarkers illustrating myocardial injury and necrosis that have been used for diagnosis include the traditional troponin levels, serum myoglobin, creatinekinase myocardial-brain isoenzyme, and the more novel heart-type fatty acid-binding protein and copeptin that can detect acute coronary syndromes (ACS) within the early hours from onset. ${ }^{5}$ Simultaneously, laboratory markers have also been used for risk prognosis assessment in complex multi-marker risk prediction tools, which can be applied before and after the acute coronary event, thus depicting the characteristics of the so-called 'vulnerable patient', who is at risk for either an initial ACS or for further complications following the acute event. . $^{5-9}$

Cardiovascular vulnerability refers not only to the presence of unstable coronary atherosclerotic plaques, but also to the vulnerable blood and vulnerable myocardium, all enmeshed into a complex, novel concept: the vulnerable patient. ${ }^{10}$ The thrombogenic vulnerable blood is characterized as presenting increased levels of inflammatory mediators (C-reactive protein, interleukins, vascular cell adhesion molecules, soluble ligand $\mathrm{CD} 40$, leukocytosis), enhanced coagulability and platelet aggregation, as well as associated hypoalbuminemia, thrombocytosis, and hyperlipidemia, and is specifically defined as prone to arterial and venous thrombosis. ${ }^{8,10,11}$

Hypoalbuminemia has been shown to associate with all-cause mortality rates, as well as with cardiovascular deaths. ${ }^{12,13}$ The serum level of albumin is determined by various factors such as synthesis and catabolic rates, ex- tracellular distribution, and exogenous elimination. Its synthesis is decreased by the interrelation between the poor nutritional status and enhanced systemic inflammation, the latter also triggering a greater catabolic rate. ${ }^{13,14}$

Therefore, the decreased nutritional status of the cardiovascular patient may poorly influence serum albumin levels and stimulate systemic inflammation, which in turn will affect blood homeostasis towards a high propensity for blood vulnerability and subsequent high cardiovascular risk. ${ }^{8,10}$ Serum albumin has been introduced in several scores that assess the overall nutritional status of subjects admitted in the hospital.

The Controlling Nutritional Status (CONUT) score has been introduced in 2005 as a tool for malnutrition screening in hospitalized subjects, and its calculation is based on three serum parameters: albumin, total cholesterol, and total lymphocyte count. ${ }^{15}$ The score represents an efficient tool for early identification and continuous control of evaluating the protein reserve, immune status, and caloric depletion in subjects admitted in the hospital for various cardiac and non-cardiac disorders, and has been associated with a high risk of complications and worse outcomes. ${ }^{16-18}$

The prognostic nutritional index (PNI) score uses 2 serum parameters: albumin and lymphocyte levels. The score reflects the immune and nutritional status and can indicate systemic inflammation, and is associated with higher mortality in surgical, medical, cardiovascular, and oncological patients. ${ }^{19-22}$

Although the negative influence of a poor nutritional status has been evaluated in previous studies on the prognosis and outcome of stable and decompensated chronic heart failure patients, ${ }^{16,21,23}$ as well as in patients with stable coronary artery disease or hypertension, ${ }^{18,24}$ the usefulness of the CONUT and PNI scores as markers for blood vulnerability on the early outcome of subjects with ST and non-ST elevation AMI is still not completely elucidated.

Therefore, the aim of the present study was to assess the correlation between nutritional status, as expressed by CONUT and PNI scores, and (1) clinical and laboratory characteristics, (2) complication rates and (3) length of hospitalization, in patients with acute myocardial infarction (STEMI and NSTEMI) admitted in an intensive cardiac care unit.

\section{MATERIALS AND METHODS}

We conducted a prospective, observational study on 56 consecutive patients with acute ST and non-ST elevation myocardial infarction admitted in the Intensive Car- 
diac Care Unit of the Cardiology Clinic of the Tîrgu Mureș County Emergency Clinical Hospital, a tertiary intensive cardiac care unit.

All patients had signed written informed consent prior to their enrollment, the study was conducted according to the principles stated in the declaration of Helsinki and was approved by the ethics committee of the institution.

All patients underwent invasive coronary angiography and coronary revascularization according to the European Society of Cardiology Guidelines for Myocardial Revascularization. ${ }^{4}$ Transthoracic echocardiography for the assessment of left ventricular function was performed on day 3 after the acute event in all patients. Blood samples were obtained for analysis of the full blood cell count, serum cholesterol and triglycerides, glycemia, serum albumin, renal function parameters (creatinine, urea), cardiac necrosis enzymes (troponin, creatine-kinase, aspartate amino transferase, alanine amino transferase, lactate dehydrogenase), and electrolyte levels (sodium, potassium).

Data regarding the demographic, clinical, and hemodynamic characteristics upon admission, as well as the cardiovascular risk factors and comorbidities were obtained from the patient medical records.

Evaluation of the nutritional status consisted in calculation of the CONUT and PNI scores in all patients. The PNI was calculated as follows: PNI $=10 \times$ serum albumin $(\mathrm{g} /$ $d L)+0.005 \times$ total lymphocyte (count per microliter). The CONUT score was calculated by using the serum levels of albumin, the total lymphocyte count, and the cholesterol levels (range $0-12$ ). In this scoring system, point values are assigned to different ranges of the laboratory measures as follows: serum albumin $\geq 3.5 \mathrm{~g} / \mathrm{dL}$, o points; $3.49-3 \mathrm{~g} / \mathrm{dL}$, 2 points; $2.99-2.5 \mathrm{~g} / \mathrm{dL}, 4$ points; and $<2.5 \mathrm{~g} / \mathrm{dL}, 6$ points; lymphocytes $\geq 1600 / \mathrm{mm}^{3}$, o points; $1200-1599 / \mathrm{mm}^{3}$, 1 point; $800-1199 / \mathrm{mm}^{3}, 2$ points; and $<800 / \mathrm{mm}^{3}, 3$ points; and total cholesterol $\geq 180 \mathrm{mg} / \mathrm{dL}$, o points; $140-179 \mathrm{mg} / \mathrm{dL}$, 1 point; 100-139 mg/dL, 2 points; and $<100 \mathrm{mg} / \mathrm{dL}$, 3 points. Patients with a CONUT score of $0-1$ have a normal nutritional status, those with a CONUT score of 2-4 are at mild risk of malnutrition, those with a CONUT score of 5-8 are at moderate risk, and those with a CONUT score of $9-12$ are at severe risk.

The study population was divided into 2 groups according to the calculated CONUT score, as follows: group 1 - CONUT score $<3$ points, specifically patients with normal to mildly impaired nutritional status, and group 2 CONUT score $\geq 3$ points, subjects with moderate to severe malnutrition.

The primary end-point of the study consisted in the rate of in-hospital complications including left ventricu- lar free wall rupture, hemodynamic instability requiring inotropic medication, high-degree atrioventricular block, the need for temporary cardiostimulation, supraventricular and ventricular arrhythmias, and in-hospital cardiac arrest. The secondary end-points included the duration of hospitalization and the length of stay in the intensive care unit.

\section{RESULTS}

The study included 56 patients with AMI (44.64\%, $\mathrm{n}=25$ with STEMI and 55.35\%, $\mathrm{n}=31$ with NSTEMI). The mean age of the study population was $61.96 \pm 13.42$ years, and $58.92 \%(n=33)$ were males.

According to the CONUT score, the study population was divided into 2 groups: group 1 included 43 patients (76.78\%) with normal to mildly depressed nutritional status and a CONUT score of $<3$ points, while group 2 included 13 patients $(23.21 \%)$ with moderate to severe malnutrition, with calculated CONUT scores of $\geq 3$ points.

The baseline characteristics of the total study population, as well as of the two CONUT groups are listed in Table 1. There were no statistically significant differences between the two CONUT groups regarding age, gender, body mass index, provenance, nor in relation to the cardiovascular risk factors of medical history of cardiovascular diseases (Table 1). The body mass index was $28.8 \pm 6.5$ $\mathrm{kg} / \mathrm{m}^{2}$ for the overall study populations, and no significant difference was recorded between the two patient groups (group $1-28.6 \pm 5.7 \mathrm{~kg} / \mathrm{m}^{2}$ vs. group $2-29.6 \pm 8.8 \mathrm{~kg} / \mathrm{m}^{2}$, $\mathrm{p}=0.87)$.

The calculated PNI for the overall population was $51.3 \pm$ 10.8 points, and the results between the two groups were in agreement with the CONUT score, as group 1 presented a significantly higher PNI score compared to group 2 (54.4 \pm 10.4 vs. $41.1 \pm 2.8, \mathrm{p}<0.0001$ ).

The clinical parameters upon admission did not significantly differ between the two groups when referred to the heart rate $(77.4 \pm 13.4$ bpm vs. $80.1 \pm 14.9 \mathrm{bpm}, \mathrm{p}=0.53)$ and systolic $(129.8 \pm 22.4 \mathrm{mmHg}$ vs. $121.4 \pm 16.8 \mathrm{mmHg}$, $\mathrm{p}=0.29)$ or diastolic $(75.7 \pm 14.5 \mathrm{mmHg}$ vs. $72.7 \pm 11.0$ $\mathrm{mmHg}, \mathrm{p}=0.57$ ) blood pressures.

The laboratory tests of the overall study population, as well as those of the two CONUT groups are listed in Table 2. No significant differences were found between the two groups regarding the renal function parameters, myocardial necrosis enzymes, or triglyceride levels. However, as expected, the serum albumin was significantly lower in patients with moderate to severe impaired nutritional status (group $1-4.1 \pm 0.3 \mathrm{~g} / \mathrm{dL}$ vs. group $2-3.6 \pm 0.3 \mathrm{~g} / \mathrm{dL}$, 
TABLE 1. Baseline characteristics of the general study population and the two CONUT groups

\begin{tabular}{|c|c|c|c|c|}
\hline Parameters & $\begin{array}{l}\text { Total } \\
\mathrm{n}=56\end{array}$ & $\begin{array}{c}\text { Group } 1-\text { CONUT }<3 \\
n=43\end{array}$ & $\begin{array}{c}\text { Group } 2-\text { CONUT } \geq 3 \\
n=13\end{array}$ & $\mathrm{p}$ value \\
\hline Male gender, n (\%) & $33(58.92 \%)$ & $26(58.13 \%)$ & $7(53.84 \%)$ & 0.67 \\
\hline Age (years, mean \pm SD) & $61.96 \pm 13.42$ & $60.58 \pm 13.76$ & $66.53 \pm 11.56$ & 0.16 \\
\hline $\mathrm{BMI}\left(\mathrm{kg} / \mathrm{m}^{2}\right.$, mean $\left.\pm \mathrm{SD}\right)$ & $28.8 \pm 6.5$ & $28.6 \pm 5.7$ & $29.6 \pm 8.8$ & 0.87 \\
\hline Rural provenance, $\mathrm{n}(\%)$ & $24(42.85 \%)$ & $18(41.86 \%)$ & $6(46.15 \%)$ & 0.78 \\
\hline Smoking status, n (\%) & $31(55.35 \%)$ & $24(55.81 \%)$ & $7(53.84 \%)$ & 0.9 \\
\hline *Obesity, n (\%) & $43(76.78 \%)$ & $32(74.41 \%)$ & $11(84.61 \%)$ & 0.44 \\
\hline MI type, STEMI n (\%) & $25(44.64 \%)$ & $28(65.11 \%)$ & $7(53.84 \%)$ & 0.46 \\
\hline MI type, NSTEMI n (\%) & $31(55.35 \%)$ & $15(34.88 \%)$ & $6(46.15 \%)$ & \\
\hline Killip class 1 & $53(94.64 \%)$ & $41(95.34 \%)$ & $12(92.30 \%)$ & 0.55 \\
\hline Killip class 2 & $3(5.35 \%)$ & $2(4.65 \%)$ & $1(7.69 \%)$ & \\
\hline Hypertension, n (\%) & $45(80.35 \%)$ & $33(76.74 \%)$ & $12(92.30 \%)$ & 0.25 \\
\hline Diabetes mellitus, n (\%) & $19(33.92 \%)$ & $16(37.20 \%)$ & $3(23.07 \%)$ & 0.34 \\
\hline Dyslipidemia, n (\%) & $17(30.35 \%)$ & $12(27.90 \%)$ & $5(38.46 \%)$ & 0.46 \\
\hline Previous angina pectoris, $\mathrm{n}(\%)$ & $12(21.42 \%)$ & $7(16.27 \%)$ & $5(38.46 \%)$ & 0.08 \\
\hline History of ischemic cardiomyopathy, $\mathrm{n}(\%)$ & $42(75 \%)$ & $30(69.76 \%)$ & $12(92.3 \%)$ & 0.10 \\
\hline Previous MI, n (\%) & $8(14.28 \%)$ & $6(13.95 \%)$ & $2(15.38 \%)$ & 0.89 \\
\hline
\end{tabular}

*Obesity defined as a calculated body mass index of over $25 \mathrm{~kg} / \mathrm{m} 2$; MI - myocardial infarction; STE - ST segment elevation MI; NSTE - non-ST elevation MI; **NYHA New York Health Association class for heart failure on admission in patients with a history of chronic heart failure.

$\mathrm{p}<0.0001$ ), as well as the total cholesterol levels (group $1-194.9 \pm 41.5 \mathrm{mg} / \mathrm{dL}$ vs. group $2-161.2 \pm 58.2 \mathrm{mg} / \mathrm{dL}$, $\mathrm{p}=0.02)$.

The analysis of the complete blood cell count showed that patients with nutritional deficit (group 2) presented lower levels of hematocrit $(\mathrm{p}=0.003)$, hemoglobin $(\mathrm{p}=$ 0.002 ), and lymphocytes ( $\mathrm{p}<0.0001)$ compared to group 1. On the other hand, malnourished subjects presented a significantly higher platelet count $(\mathrm{p}=0.001)$, a higher mean platelet volume (MPV) $(\mathrm{p}=0.03)$, as well as more increased neutrophil/lymphocyte $(\mathrm{p}<0.0001)$ and platelet/lymphocyte $(\mathrm{p}<0.0001)$ ratios, indicating enhanced blood thrombogenicity and inflammation (Table 2).

The primary end-point of the study consisting in the rate of complications that had occurred during hospitalization is illustrated in Figure 1. In total, 17.8\% $(n=10)$ of subjects were hemodynamically unstable requiring inotropic therapy, $1.78 \%(\mathrm{n}=1)$ presented high-degree atrioventricular block which required the use of a temporary pacemaker, $12.5 \%(\mathrm{n}=7)$ and $7.14 \%(\mathrm{n}=4)$ presented ventricular and supraventricular arrhythmias respectively, while $5.35 \%(n=3)$ of patients presented in-hospital cardiac arrest (Figure 1A). When comparing the two CONUT groups, there were no statistically significant differences between the two regarding the rate of supraventricular ( $\mathrm{p}$ $=0.93)$ or ventricular arrhythmias $(\mathrm{p}=0.54)$, high-degree atrioventricular block $(\mathrm{p}=0.57)$ or the need for temporary pacemaker $(p=0.57)$, or of in-hospital cardiac arrest ( $p$
$=0.57)$. However, the results showed that $38.4 \%$ of patients with moderate to severe nutritional impairment had presented hemodynamic instability and required inotropic medication, compared to only $11.6 \%$ of patients with normal or mildly depressed nutritional status $(\mathrm{p}=0.02)$ (Figure 1B).

Analysis of the secondary end-point of the study showed an overall length of hospital stay of $8.3 \pm 2.8$ days, out of which $3.01 \pm 1.4$ were in the Intensive Cardiac Care Unit. The group comparison according to the CONUT score found that the subjects in group 2 required a significantly higher period of admission in the Intensive Cardiac Care Unit, compared to the group with good nutritional status ( $\mathrm{p}=$ 0.02) (Figure 2A) and a slightly longer period of stay in the hospital in terms of total hospitalization days, however, without statistical significance $(\mathrm{p}=0.06)$ (Figure $2 \mathrm{~B})$.

\section{DISCUSSIONS}

The present study primarily aimed to identify the relationship between an impaired nutritional status and inhospital complications, as well as with the clinical profile and the hospitalization period in patients with AMI who were admitted in a tertiary intensive cardiac care unit.

The nutritional status of the patients has been recently studied as having significant impact on prognosis, in a sense that malnutrition can negatively impact patient outcomes in various chronic disorders, from oncological 
TABLE 2. Baseline characteristics of the general study population and the two CONUT groups

\begin{tabular}{|c|c|c|c|c|}
\hline Parameter & $\begin{array}{l}\text { Total } \\
\mathrm{n}=56\end{array}$ & $\begin{array}{c}\text { Group } 1-\text { CONUT }<3 \\
n=43\end{array}$ & $\begin{array}{c}\text { Group } 2-\text { CONUT } \geq 3 \\
n=13\end{array}$ & $\mathrm{p}$ value \\
\hline & Mean \pm SD $(95 \%$ CI $)$ & Mean \pm SD $(95 \% \mathrm{CI})$ & Mean \pm SD $(95 \% \mathrm{CI})$ & \\
\hline \multicolumn{5}{|l|}{ Biochemical laboratory tests } \\
\hline Uric acid (mg/dL) & $5.1 \pm 1.6(4.4-5.8)$ & $5.3 \pm 1.5(4.5-6.1)$ & $4.3 \pm 1.7(2.1-6.5)$ & 0.23 \\
\hline Urea (g/dL) & $48.6 \pm 52.6(34.5-62.7)$ & $48.2 \pm 57.7(30.4-66.0)$ & $49.8 \pm 32.2(30.3-69.3)$ & 0.27 \\
\hline Creatinine (g/dL) & $1.6 \pm 2.8(0.8-2.3)$ & $1.7 \pm 3.2(0.8-2.7)$ & $1.0 \pm 0.5(0.7-1.4)$ & 0.6 \\
\hline Sodium (mmol/L) & $140.4 \pm 5.8(138.8-142.0)$ & $139.72 \pm 2.9(138.8-140.6)$ & $142.9 \pm 10.9(136.3-149.5)$ & 0.89 \\
\hline Potassium (mmol/L) & $4.2 \pm 0.5(4.1-4.3)$ & $4.2 \pm 0.5(4.0-4.3)$ & $4.3 \pm 0.4(4.0-4.6)$ & 0.57 \\
\hline Albumin (g/dL) & $4.0 \pm 0.3(3.9-4.1)$ & $4.1 \pm 0.3(4.0-4.2)$ & $3.6 \pm 0.3(3.4-3.7)$ & $<0.0001$ \\
\hline Total cholesterol (mg/dL) & $187.1 \pm 47.6(174.3-199.8)$ & $194.9 \pm 41.5(182.1-207)$. & $161.2 \pm 58.2(126.0-196.4)$ & 0.02 \\
\hline Triglycerides (mg/dL) & $198.0 \pm 172.3(117.4-278.6)$ & $186.4 \pm 108.0(126.6-246.2)$ & $187.5 \pm 245.4(-17.7-392.7)$ & 0.05 \\
\hline Glycemia (mg/dL) & $135.1 \pm 53.1(120.8-149.3)$ & $140.4 \pm 55.7(123.2-157.5)$ & $117.4 \pm 40.3(93.0-141.8)$ & 0.21 \\
\hline Troponin (ng/mL) & $1.42 \pm 2.4(0.7-2.1)$ & $1.51 \pm 2.6(0.6-2.3)$ & $1.09 \pm 1.70(-0.04-2.2)$ & 0.44 \\
\hline CK $(\mathrm{U} / \mathrm{L})$ & $1,459 \pm 1,773(979.5-1,938)$ & $1,433 \pm 1,716(898.5-1,968)$ & $1,541 \pm 2,018(321.8-2,760)$ & 0.89 \\
\hline ASAT (U/L) & $167.9 \pm 187.5(117.2-218.7)$ & $164.3 \pm 186.8(106.1-222.5)$ & $179.9 \pm 197.1(60.8-299)$ & 0.75 \\
\hline ALAT (U/L) & $62.7 \pm 70.5(43.4-81.9)$ & $55.5 \pm 45.2(41.2-69.8)$ & $85.4 \pm 120(12.8-157.9)$ & 0.59 \\
\hline $\mathrm{LDH}(\mathrm{U} / \mathrm{L})$ & $617.2 \pm 469.6(490.2-744.3)$ & $584.3 \pm 442.8(446.4-722.3)$ & $723.7 \pm 553.9(1389-1058)$ & 0.3 \\
\hline \multicolumn{5}{|l|}{ Complete blood cell count } \\
\hline Hematocrit (\%) & $40.9 \pm 7.4(38.9-43.0)$ & $41.8 \pm 7.2(39.5-44.1)$ & $38.1 \pm 7.6(33.5-42.8)$ & 0.003 \\
\hline Hemoglobin (mg/dL) & $14.0 \pm 1.9(13.5-14.5)$ & $14.4 \pm 1.4(13.9-14.9)$ & $12.6 \pm 2.5(11.1-14.1)$ & 0.002 \\
\hline Neutrophil count (\%) & $64.0 \pm 20.9(58.4-69.6)$ & $61.7 \pm 20.0(55.5-67.8)$ & $71.7 \pm 22.8(57.9-85.5)$ & 0.13 \\
\hline Leucocytes count $(/ \mu \mathrm{L})$ & $13,568 \pm 17,285(8940-18197)$ & $14,371 \pm 19,658(83.23-20419)$ & $10,913 \pm 2,765(9,242-12,584)$ & 0.7 \\
\hline Thrombocyte count $(/ \mu \mathrm{L})$ & $\begin{array}{l}279,738 \pm 209,746 \\
(223,567-335,908)\end{array}$ & $\begin{array}{c}212,776 \pm 79,337 \\
(212,776-261,610)\end{array}$ & $\begin{array}{l}420,461 \pm 389,443 \\
(185,124-655,799)\end{array}$ & 0.001 \\
\hline Lymphocyte count $(/ \mu \mathrm{L})$ & $2,246 \pm 1,859(1748-2744)$ & $2,619 \pm 1,970(2012-3225)$ & $1,013 \pm 331.3(813.2-1,214)$ & $<0.0001$ \\
\hline Neutrophil/Lymphocyte ratio & $0.04 \pm 0.04(0.03-0.05)$ & $0.03 \pm 0.02(0.02-0.04)$ & $0.08 \pm 0.06(0.01-0.12)$ & $<0.0001$ \\
\hline Platelet/Lymphocyte ratio & $198.3 \pm 245.3(132.6-264.0)$ & $116.4 \pm 70.35(94.7-138.0)$ & $469.4 \pm 393.5(231.6-707.2)$ & $<0.0001$ \\
\hline RDW-Platelet ratio & $0.07 \pm 0.10(0.05-0.10)$ & $0.07 \pm 0.10(0.04-0.10)$ & $0.09 \pm 0.09(0.03-0.14)$ & 0.32 \\
\hline RDW-DS (fL) & $47.1 \pm 5.3(44.8-49.5)$ & $47.1 \pm 5.4(44.0-50.1)$ & $47.3 \pm 5.6(43.6-52.0)$ & 0.91 \\
\hline Mean platelet volume (\%) & $0.23 \pm 0.07(0.20-0.26)$ & $0.21 \pm 0.06(0.18-0.24)$ & $0.28 \pm 0.07(0.21-0.36)$ & 0.03 \\
\hline PDW (fL) & $15.2 \pm 3.3(14.0-16.5)$ & $15.3 \pm 3.3(13.8-16.8)$ & $13.5 \pm 3.3(10.6-16.3)$ & 0.18 \\
\hline
\end{tabular}

to cardiovascular disease, and from chronic heart failure to stable coronary artery disease, myocardial infarction, or lower limb ischemia. ${ }^{16-24}$

Evaluation of the nutritional status could become a new prognostic marker for such patients, while dietary supplements and malnutrition corrective therapies may beneficially influence the prognosis of patients with chronic heart failure. ${ }^{25,26}$ The CONUT score (Controlling Nutritional Status), based on the levels of serum albumin, total cholesterol, and lymphocyte count, has been developed as a simple, cost-efficient screening tool for impaired nutrition in hospitalized subjects. The CONUT index classifies the nutritional status into normal, lightly decreased, moderate, and severely impaired. ${ }^{15}$

Serum albumin, also included in the CONUT score, is a multifunctional human protein influenced by various fac- tors, including the nutritional status and enhanced systemic inflammation. Furthermore, low serum albumin has also been linked to all-cause mortality and cardiovascular mortality and was associated with malnutrition, a pro-inflammatory and a pro-thrombotic status, all accounting for the definition of vulnerable blood, as a part of a larger concept: the vulnerable cardiovascular patient. ${ }^{8,13}$ The results of the current investigation also found low levels of albumin in undernourished patients with AMI. Concomitantly, subjects with malnutrition and AMI in our study presented lower serum cholesterol levels compared to controls, which could further influence lipid-lowering therapies, as hypocholesterolemia has been previously reported to negatively impact mortality in the general population. ${ }^{27-29}$

The lower lymphocyte count has been shown to lead to worse outcomes in subjects with coronary artery disease. 

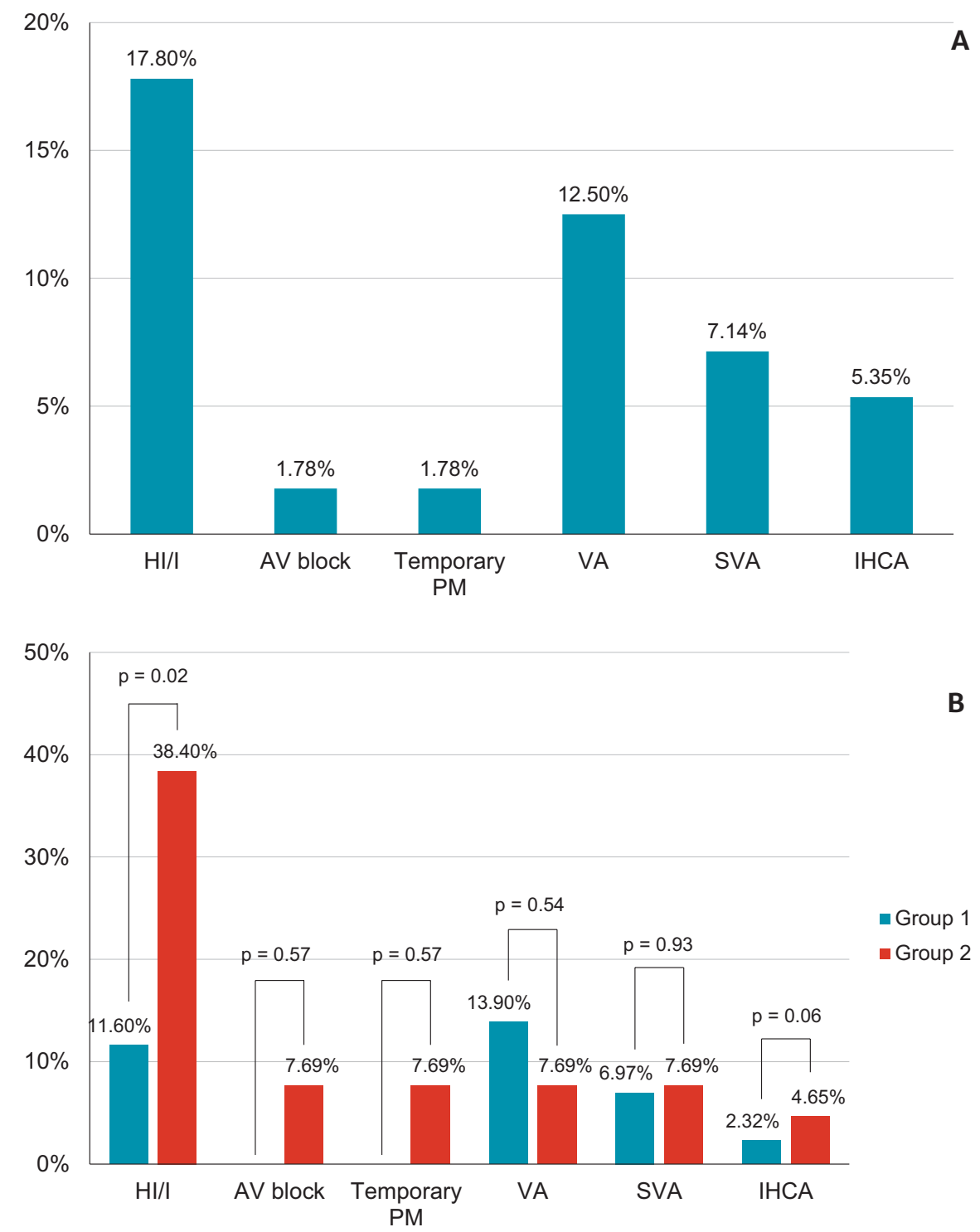

FIGURE 1. The rate of complications during hospitalization (A) for the overall study population; (B) for the CONUT groups. HI/I - hemodynamic instability or inotropic requirement; $A V$ block - high-degree atrioventricular block; temporary PM - temporary pacemaker; VA - ventricular arrhythmias; SVA - supraventricular arrhythmias; IHCA - inhospital cardiac arrest

In a study conducted by Shiyovich et al., it was revealed that the lymphocyte count, the neutrophil-to-lymphocyte ratio, and the eosinophil count were independently associated with long-term mortality following an AMI.. ${ }^{30}$ Similarly, our results indicated a significantly lower lymphocyte count and a higher neutrophil/lymphocyte count in patients with impaired nutritional status, who also presented a higher rate of in-hospital complications.

The findings of the present study indicated that patients with moderate to severe impairment of the nutritional status, assessed with the CONUT index, are at in- creased risk of presenting hemodynamic instability and thus requiring inotropic therapy. Similarly, patients with a CONUT score of more than 3 points, were more likely to present rhythm or conduction abnormalities, as well as life-threatening complications such as in-hospital cardiac arrest, but the results showed no statistical significance, most likely due to the low number of patients enrolled in the study.

Other studies have evaluated the prognostic impact of the nutritional status on admission in myocardial infarction patients. Basta et al. evaluated the influence of the CO- 

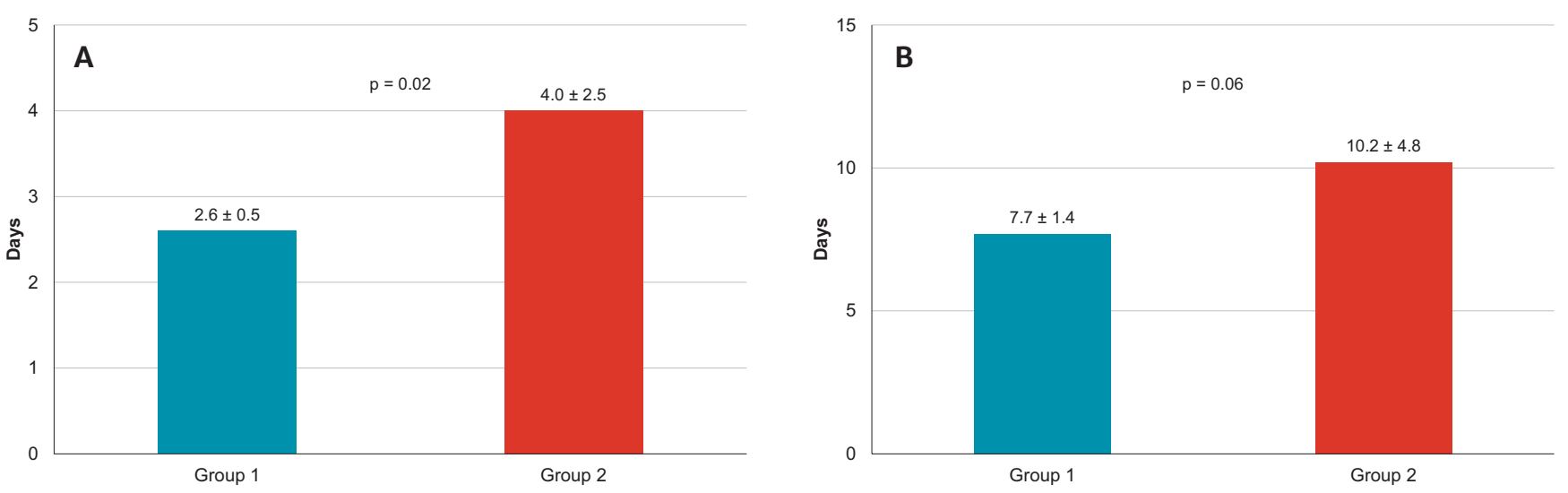

FIGURE 2. Duration of hospitalization in the study groups. A - Length of stay in the Intensive Cardiac Care Unit (days). B - Total number of hospitalization days

NUT score and of the PNI on the outcome of 945 patients with STEMI, on a median follow-up period of 2 years, and concluded that nutritional status, in association with other risk factors, leads to an increased mortality, and that the nutritional indexes correlate with other markers for poor outcomes, including renal function parameters, natriuretic peptide, and transaminases. ${ }^{22}$ Another study by Keskin et al. also evaluated the impact of malnutrition in 1,823 STEMI patients on in-hospital mortality and longterm prognosis. Their results were concordant with those of the present study, in the way that undernutrition, evaluated by the PNI (based on albumin levels and lymphocyte count), was significantly associated with higher mortality, after adjustments for all confounders. ${ }^{19}$

Having drawn increasing attention, the nutritional status of hospitalized patients has been in the focus of many cardiovascular studies, most frequently regarding its influence in heart failure. Iwakami et al. measured the CONUT score in 635 acute heart failure patients and found that over a follow-up time of 324 days, the nutritional scoring was independently associated with the death rate (c-statistic of $0.71, \mathrm{p}<0.001$ ). ${ }^{16}$ Shirakabe et al. found similar results when studying the predictive capacity of the PNI and CONUT indexes on the 365-day mortality of 458 patients hospitalized in the intensive care unit for acute heart failure (HR 2.060, 95\% CI: $1.302-3.259$ for PNI; HR 2.238, 95\%CI: 1.050-4.772 for CONUT). ${ }^{23}$ The present study focused on patients with AMI, and one of the major end-points consisted in the development of acute heart failure secondary to the acute ischemic event, which has been incompletely studied. Our results proved that subjects with malnutrition are more likely to develop acute heart failure and hemodynamic instability compared to patients with normal or mildly decreased CONUT score.
Coronary artery disease severity and prognosis have also been reported by various studies in which the CONUT score was found to be an independent predictor of longterm adverse cardiac events. ${ }^{24,31}$ Furthermore, it appears that the CONUT index can predict short and mid-term all-cause mortality in hypertensive patients, as reported by Sun et al., thus suggesting that assessment of the nutritional state could offer supplementary evidence in subjects with increased blood pressure. ${ }^{18}$

Regarding the hospitalization time, the results of the present study revealed that malnourished patients required both a longer observation time in the intensive cardiac care unit, as well as a longer overall hospital stay. This could be explained by the higher rate of complications and hemodynamic instability, which led to an increased need for continuous monitoring and intensive therapy with vasoactive or interventional therapies (temporary pacemaker). A multicenter study conducted by Kang et al. in 25 hospitals in Korea, found that patients with malnutrition had a longer hospital stay and a lower survival rate during the 90-day follow-up. ${ }^{32}$ Agarwall et al. also reported that subjects with a low nutritional status presented a greater median length of hospital stay and a significantly higher rate of readmissions. ${ }^{33}$ Consequently, there is a need for implementation of a screening program for nutritional statuses, as well as an interdisciplinary approach together with a nutrition specialist in case of hospitalized patients, in order to provide active management of malnutrition, which may improve outcomes both on short and long term.

\section{STUDY LIMITATIONS AND FUTURE DIRECTIONS}

The main limitation of the study was that it was a singlecenter, observational one, with a relatively low number of 
enrolled subjects, and the results could have been influenced by unmeasurable variables. Secondly, the study did not include all-cause mortality and cardiovascular mortality as a primary end-point, which is explained, again, by the low number of patients and the absence of any death during hospitalization.

The future direction of the present research is to add the analysis of serum biomarkers that express an enhanced inflammatory status, as a part of the complete representation of vulnerable blood in patients with acute coronary syndromes. Another direction of the study could be to assess ventricular remodeling and the rate of major adverse cardiovascular events in relation to the nutritional status of AMI patients.

\section{CONCLUSIONS}

This study proved that nutritional deficit is associated with an increased rate of complications during hospital stay in patients with AMI. Patients with a CONUT score higher than 3 points (moderate to severe malnutrition) presented an increased rate of acute heart failure with hemodynamic instability and requirement of inotropic therapy and required longer observation times in the intensive cardiac care unit, compared to subjects with a CONUT score of $<3$ points (normal to mildly impaired nutritional status). The screening of malnutrition in patients hospitalized for AMI could lead to decreased in-hospital adverse events, shorter admission periods, and consequently lower healthcare costs.

\section{CONFLICT OF INTEREST}

Nothing to declare.

\section{ACKNOWLEDGEMENT}

The present research is receiving funding from the University of Medicine and Pharmacy of Tîrgu Mureș, Romania, via governmental funds for PhD studies, contract number 13406/3/07.10.2015.

\section{REFERENCES}

1. Thygesen K, Alpert JS, Jaffe AS, et al. Third Universal Definition of Myocardial Infarction. Circulation. 2012;126:2020-2035. https://doi.org/10.1161/CIR.ob013e31826e1058.

2. Ibanez B, James S, Agewall S, et al. 2017 ESC Guidelines for the management of acute myocardial infarction in patients presenting with ST-segment elevation: The Task Force for the management of acute myocardial infarction in patients presenting with ST-segment elevation of the European Society of Cardiology (ESC). Eur Heart J. 2018;39:119-177. doi: 10.1093/eurheartj/ehx393.

3. Roffi M, Patrono C, Collet JP, et al. 2015 ESC Guidelines for the management of acute coronary syndromes in patients presenting without persistent ST-segment elevation: Task Force for the Management of Acute Coronary Syndromes in Patients Presenting without Persistent ST-Segment Elevation of the European Society of Cardiology (ESC). Eur Heart J. 2016;37:267-315. doi: 10.1093/eurheartj/ehv320.

4. Kolh P, Windecker S, Alfonso F, et al. 2014 ESC/EACTS Guidelines on myocardial revascularization: the Task Force on Myocardial Revascularization of the European Society of Cardiology (ESC) and the European Association for CardioThoracic Surgery (EACTS). Developed with the special contribution of the European Association of Percutaneous Cardiovascular Interventions (EAPCI). Eur J Cardiothorac Surg. 2014;46:517-592. doi: 10.1093/ejcts/ezu366.

5. Chan D, Ng LL. Biomarkers in acute myocardial infarction. BMC Medicine. 2010;8:34. doi:10.1186/1741-7015-8-34.

6. Waxman S, Ishibashi F, Muller JE. Detection and Treatment of Vulnerable Plaques and Vulnerable Patients Novel Approaches to Prevention of Coronary Events. Circulation. 2006;114:23902411. doi: 10.1161/CIRCULATIONAHA.105.540013.

7. Narain VS, Gupta N, Sethi R, et al. Clinical correlation of multiple biomarkers for risk assessment in patients with acute coronary syndrome. Indian Heart J. 2008;60:536-542.

8. Naghavi M, Libby P, Falk E, et al. From vulnerable plaque to vulnerable patient: a call for new definitions and risk assessment strategies: Part II. Circulation. 2003;108:17721778. doi: 10.1161/01.CIR.0000087481.55887.C9.

9. Tello-Montoliu A, Marín F, Roldán V, et al. A multimarker risk stratification approach to non-ST elevation acute coronary syndrome: implications of troponin T, CRP, NT pro-BNP and fibrin D-dimer levels. J Intern Med. 2007;262:651-658. doi: 10.1111/j.1365-2796.2007.01871.x.

10. Naghavi M, Libby P, Falk E, et al. From vulnerable plaque to vulnerable patient: a call for new definitions and risk assessment strategies: Part I. Circulation. 2003;108:16641672. doi: 10.1161/01.CIR.0000087480.94275.97.

11. Hess K, Marx N, Lehrke M. Cardiovascular disease and diabetes: the vulnerable patient. Eur Heart J Supplements. 2012;14:B4-B13. doi:10.1093/eurheartj/sus002.

12. Danesh J, Collins R, Appleby P, Peto R. Association of fibrinogen, C-reactive protein, albumin, or leukocyte count with coronary heart disease: meta-analyses of prospective studies. JAMA. 1998; 279:1477-1482.

13. Chien SC, Chen CY, Lin CF, Yeh HI. Critical appraisal of the role of serum albumin in cardiovascular disease. Biomarker Research. 2017;5:31. doi:10.1186/s40364-017-0111-X.

14. Don BR, Kaysen G. Serum albumin: relationship to inflammation and nutrition. Semin Dial. 2004;17:432-437. doi: 10.1111/j.0894-0959.2004.17603.X.

15. Ignacio de Ulibarri J, Gonzalez-Madrono A, de Villar NG, et al. CONUT: a tool for controlling nutritional status. First validation in a hospital population. Nutr Hosp. 2005;20:38-45.

16. Iwakami N, Nagai T, Furukawa TA, et al. Prognostic value of malnutrition assessed by Controlling Nutritional Status score for long-term mortality in patients with acute heart failure. Int J Cardiol. 2017;230:529-536. doi: 10.1016/j.ijcard.2016.12.064. 
17. Toyokawa T, Kubo N, Tamura $\mathrm{T}$, et al. The pretreatment Controlling Nutritional Status (CONUT) score is an independent prognostic factor in patients with resectable thoracic esophageal squamous cell carcinoma: results from a retrospective study. BMC Cancer. 2016;16:722. doi:10.1186/ s12885-016-2696-0.

18. Sun X, Luo L, Zhao X, Ye P. Controlling Nutritional Status (CONUT) score as a predictor of all-cause mortality in elderly hypertensive patients: a prospective follow-up study. BMJ Open. 2017;7:e015649. doi: 10.1136/bmjopen-2016-015649.

19. Keskin M, Hayıroğlu Mİ, Keskin T, et al. A novel and useful predictive indicator of prognosis in ST-segment elevation myocardial infarction; prognostic nutritional index. Nutr Metab Cardiovasc Dis. 2017;27:438-446. doi: 10.1016/j. numecd.2017.01.005.

20. Yang L, Xia L, Wang Y, et al. Low Prognostic Nutritional Index (PNI) Predicts Unfavorable Distant Metastasis-Free Survival in Nasopharyngeal Carcinoma: A Propensity Score-Matched Analysis. PLoS One. 2016;11:e0158853. doi: 10.1371/journal. pone.0158853.

21. Narumi T, Arimoto T, Funayama A, et al. Prognostic importance of objective nutritional indexes in patients with chronic heart failure. J Cardiol. 2013;62:307-313. doi: 10.1016/j. jjcc.2013.05.007.

22. Basta G, Chatzianagnostou K, Paradossi U, et al. The prognostic impact of objective nutritional indices in elderly patients with ST-elevation myocardial infarction undergoing primary coronary intervention. Int J Cardiol. 2016;221:987992. doi: 10.1016/j.ijcard.2016.07.039.

23. Shirakabe A, Hata N, Kobayashi N, et al. The prognostic impact of malnutrition in patients with severely decompensated acute heart failure, as assessed using the Prognostic Nutritional Index (PNI) and Controlling Nutritional Status (CONUT) score. Heart Vessels. 2018;33:134-144. doi: 10.1007/s00380-017-1034-z.

24. Wada H, Dohi T, Miyauchi K, et al. Prognostic impact of nutritional status assessed by the Controlling Nutritional

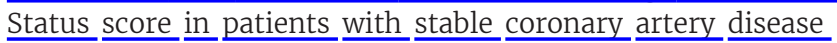
undergoing percutaneous coronary intervention. Clin Res Cardiol. 2017;106:875-883. doi: 10.1007/s00392-017-1132-z.
25. Bourdel-Marchasson I, Emeriau JP. Nutritional strategy in the management of heart failure in adults. Am J Cardiovasc Drugs. 2001;1:363-373.

26. Broqvist M, Arnqvist H, Dahlström U, Larsson J, Nylander E, Permert J. Nutritional assessment and muscle energy metabolism in severe chronic congestive heart failure effects of long-term dietary supplementation. Eur Heart J. 1994;15:1641-1650.

27. Iseki $K$, Yamazato $M$, Tozawa $M$, Takishita S. Hypocholesterolemia is a significant predictor of death in a cohort of chronic hemodialysis patients. Kidney Int. 2002;61:1887-1893. doi: 10.1046/j.1523-1755.2002.00324.X.

28. Tuikkala P, Hartikainen S, Korhonen MJ, et al. Serum total cholesterol levels and all-cause mortality in a home-dwelling elderly population: a six-year follow-up. Scandinavian Journal of Primary Health Care. 2010;28:121-127. doi: 10.3109/02813432.2010.487371.

29. Pekkanen J, Nissinen A, Vartiainen E, Salonen JT, Punsar $\mathrm{S}$, Karvonen MJ. Changes in serum cholesterol level and mortality: a 30-year follow-up. The Finnish cohorts of the seven countries study. Am J Epidemiol. 1994;139:155-165.

30. Shiyovich A, Gilutz H, Plakht Y. White Blood Cell Subtypes Are Associated with a Greater Long-Term Risk of Death after Acute Myocardial Infarction. Texas Heart Institute Journal. 2017;44:176-188. doi:10.14503/THIJ-16-5768.

31. Kunimura A, Ishii $\mathrm{H}$, Uetani $\mathrm{T}$, et al. Impact of nutritional assessment and body mass index on cardiovascular outcomes in patients with stable coronary artery disease. Int J Cardiol. 2017;230:653-658. doi:10.1016/j.ijcard.2017.01.008.

32. Kang MC, Kim JH, Ryu SW, et al. Prevalence of Malnutrition in Hospitalized Patients: a Multicenter Cross-sectional Study. Journal of Korean Medical Science. 2018;33:e10. doi:10.3346/ jkms.2018.33.e10.

33. Agarwal E, Ferguson $M$, Banks $M$, et al. Malnutrition and poor food intake are associated with prolonged hospital stay, frequent readmissions, and greater in-hospital mortality: results from the Nutrition Care Day Survey 2010. Clin Nutr. 2013;32:737-475. doi: 10.1016/j.clnu.2012.11.021. 